\title{
Tecnologias assistivas: aspectos que influenciam na assiduidade e no abandono dos recursos
}

\section{Assistive technologies: aspects influencing assurance and abandonment of resources}

(D) Marciane Montagner Missio ${ }^{1}$, Luise Ferreira de Queiroz ${ }^{2}$

\section{RESUMO}

As tecnologias assistivas são recursos utilizados para potencializar habilidades funcionais e proporcionar autonomia e independência. Pessoas com deficiência possuem a garantia de acesso a esses recursos, com prescrição de profissionais dos serviços de reabilitação do Sistema Único de Saúde (SUS). Objetivo: Verificar aspectos envolvidos com a adesão e abandono de órteses, próteses e cadeiras de rodas. Método: Trata-se de um estudo transversal, os dados foram coletados através de uma entrevista estruturada que questiona o processo de prescrição, benefícios e continuidade ou abandono de uso dos equipamentos. A população do estudo foi de indivíduos de 18 a 80 anos que receberam tecnologias assistivas dispensadas no período de janeiro a dezembro de 2017 através de um Centro Especializado em Reabilitação, município de Santa Maria-RS. Resultados: Em 2017 foram dispensadas 400 equipamentos, sendo entrevistadas 31 pessoas desse quantitativo, pois muitos dos beneficiados não residem no município, não consentindo em participar. As 31 pessoas receberam 38 equipamentos, sendo 6 cadeiras motorizadas, 13 manuais, 8 próteses de membro inferior, 1 de membro superior, 7 órteses de membro inferior e 3 de membro superior. Destes equipamentos, 20 continuam sendo utilizados e 18 foram abandonados. Os benefícios mais relatados foram a melhor estabilidade e a facilitação do deslocamento propiciada pelos recursos. Conclusão: A maioria dos beneficiários foram adultos e idosos, sendo que a cadeira manual e a prótese de membro inferior foram os equipamentos mais dispensados. Foi verificado que houve significativo número de abandono sugerindo que ocorreram inadequações no momento da prescrição, resultando no abandono.

Palavras-chave: Aparelhos Ortopédicos, Membros Artificiais, Cadeiras de Rodas, Terapia por Exercício, Terapia Ocupacional

\section{ABSTRACT}

Assistive Technologies are resources used to enhance functional abilities and provide autonomy and independence. Persons with disabilities are guaranteed access to these resources, with prescription from professionals in the rehabilitation services of the Unified Health System (SUS). Objective: Check aspects involved with adhesion and abandonment of orthoses, prostheses and wheelchairs. Method: This was a cross-sectional study, the data were collected through a structured interview that questions the prescription process, benefits and continuity or abandonment of use of the equipment's. The study population were individuals aged 18 to 80 years who received assistive technologies dispensed in the period from January to December of 2017 through a Specialized Center in Rehabilitation, of the municipality of Santa Maria - RS. Results: In 2017,400 equipment were dispensed, being interviewed 31 people of this amount, since many of the beneficiaries do not reside in the municipality, not consenting to participate. The 31 people received 38 equipment, 6 motorized chairs, 13 manuals, 8 lower limb prostheses, 1 upper limb, 7 lower limb orthoses and 3 upper limbs. Of these, 20 were still used and 18 were abandoned. The most reported benefits were improved stability and facilitation of resource displacement. Conclusion: The majority of the beneficiaries were adults and the elderly, and the manual chair and the lower limb prosthesis were the most dispensed equipment. It was verified that there was a significant number of abandonment suggesting that inadequacies occurred at the moment of prescription, resulting in the abandonment.

Keywords: Orthotic Devices, Artificial Limbs, Wheelchairs, Exercise Therapy, Occupational Therapy 


\section{INTRODUÇÃO}

A Organização Mundial de Saúde (OMS) considera que o conceito de deficiência está relacionado com muitas dificuldades e barreiras que impedem a participação igualitária das pessoas na sociedade e dificultam sua vida. ${ }^{1}$

Os dados estatísticos das Organizações das Nações Unidas (ONU) enfatizam que aproximadamente $15 \%$ da população mundial tem algum tipo de deficiência, através do último censo do Instituto Brasileiro de Geografia e Estatística (IBGE) realizado em 2010, 45 milhões dos brasileiros incluídos nessa pesquisa foram considerados indivíduos com deficiência, o que representa um grande quantitativo de pessoas com algum comprometimento físico, visual, auditivo ou intelectual. ${ }^{2}$

Historicamente, as pessoas com algum tipo de deficiência, já foram considerados como seres anormais, monstruosos, resultados de ira ou de milagres das divindades. Com o passar dos anos, os conceitos foram desmistificando-se até atingir uma melhor aceitação da sociedade, como indivíduos com alguma deficiência e detentor de direitos fundamentais garantidos por legislações como todos os outros cidadãos. $^{3}$

Algumas legislações estão em vigência, como a Lei 13.146 de 2015, denominada de Lei brasileira de Inclusão da pessoa com deficiência, para garantir direitos a igualdade de oportunidades, a educação, a habilitação e reabilitação, a aquisição de materiais ou recursos necessários para o processo de tratamento e aquisição de maior autonomia. ${ }^{4}$

As pessoas que possuem deficiência, conforme a Classificação Internacional de Funcionalidade, Incapacidade e Saúde (CIF), tendem a apresentar alterações em três áreas: na estrutura e função corporal, limitação na execução de atividades e restrição a participação social, tratando-se de um conjunto de dificuldades que interagem ou deveriam interagir adequadamente entre si. ${ }^{1}$

0 tratamento à pessoa com deficiência tem por finalidade o desenvolvimento das capacidades, habilidades e aptidões físicas, sensoriais, psicossociais, entre outras que contribuam para o alcance de sua autonomia e de condições dignas para aquisição das necessidades básicas. ${ }^{4}$ Como recurso importante e pertinente em inúmeras situações, as tecnologias assistivas são muito utilizadas para potencializar as habilidades funcionais dos indivíduos e favorecer maior autonomia e independência.

As tecnologias assistiva (TA) são um conjunto de recursos e serviços que por meios tecnológicos auxiliam pessoas com limitações funcionais, incapacidade ou mobilidade reduzida, contribuindo para prevenir agravos, proporcionar e ampliar habilidades das pessoas, promover vida independente e inclusão, pois múltiplos equipamentos podem ser criados com o propósito de melhorar a qualidade de vida e a simplificação no desempenho de determinadas funções. ${ }^{5}$

Grande parte dos serviços de reabilitação as pessoas com deficiência, são compostos por profissionais terapeutas ocupacionais, fisioterapeutas, fonoaudiólogos, psicólogos e assistentes sociais.

Tais profissionais possuem a responsabilidade em realizar intervenções terapêuticas conforme cada quadro clínico, propondo exercícios, treinamento, estratégias de compensação, disponibilização de recursos de tecnologias assistivas, apoio, aconselhamento e adequação do ambiente domiciliar. ${ }^{1}$

Mesmo com garantias de direitos para aquisição desses equipamentos, e os benefícios serem amplamente divulgados, alguns indivíduos não fazem uso desses equipamentos devido à substituição por outro recurso ou má adaptação ao dispositivo. ${ }^{6}$

Um estudo da Universidade de Poitiers na França, aplicado com 549 pessoas mostrou que o abandono de TAs pode ocorrer devido ao uso desses recursos projetarem maior evidência da presença de deficiência, não permitindo que o sujeito consiga passar despercebido ao estar em uso de uma Tecnologia deste tipo, atuando como um dos motivos para abandono dos recursos. ${ }^{7}$
Estudo italiano relacionado ao abandono de $\mathrm{TA}$, refere que o abandono é um conceito dependente de variáveis pessoais e relacionadas ao recurso, sendo as variáveis pessoais a idade, sexo, acesso à educação, dificuldade de aceitação da deficiência, imaturidade emocional, e as variáveis relacionada ao recurso, a qualidade e aparência do equipamento. ${ }^{8}$

O conceito de abandono faz referência a desistir, largar ou renunciar algo. Em contrapartida o conceito de uso significa estar acostumado ou ter hábito de praticar determinada situação. ${ }^{9} \mathrm{Em}$ pesquisa desenvolvida nos Estados Unidos, os conceitos de uso e abandono das TAs foram consideradas com algumas variações, sendo o uso parcial para quando o recurso é usado em algumas partes do tempo e outras não, uso otimizado para quando está sob todas condições recomendadas de usabilidade, uso relutante para situação em que o usuário utiliza os equipamentos com desagrado, e por último, o abandono significando renúncia permanente por frustração ou aborrecimento. ${ }^{10}$

O uso ou o abandono das TAs são temáticas de interesse, visto que são tecnologias que requerem emprego financeiro de recursos. Tratando-se de serviços do Sistema Único de Saúde, o gasto empregado em tecnologias que são abandonadas, além de evidenciarem falhas no processo de prescrição, levam a desperdício dos recursos públicos. ${ }^{11}$

Ressaltado por estudo norte americano que o indivíduo a utilizar uma tecnologia, além de participar da escolha do mais aceitável, precisa ser avaliado também seu ambiente de uso, considerando-se que quando não for de interesse do usuário, possivelmente não terá uso contínuo, sendo abandonado. ${ }^{10}$

Considerando-se que os dispositivos prescritos por serviços de reabilitação representam alto custo ao sistema público de saúde, faz se importante estudar aspectos relacionados com a prescrição, uso contínuo ou abandono dos equipamentos, a fim de compreender a efetividade no processo de prescrição e a satisfação das necessidades do paciente com o uso dos dispositivos.

\section{OBJETIVO}

O presente estudo teve por objetivo verificar aspectos envolvidos com a adesão e abandono das tecnologias assistivas, tendo como referência um serviço de reabilitação que dispensa esses recursos como órteses, próteses e cadeiras de rodas.

Foram selecionados os três recursos acima por serem amplamente prescritos no serviço de reabilitação pesquisado, de modo que pudesse gerar uma amostra representativa para o estudo.

\section{MÉTODO}

Trata-se de um estudo transversal, com abordagem quantitativa, sendo realizado um levantamento de dados por meio de uma entrevista estruturada com os participantes. A entrevista foi formulada pelas próprias pesquisadoras, buscando caracterizar a amostra, identificar aspectos sobre a forma de prescrição, os benefícios esperados, as características do recurso recebido e a atual situação de abandono ou uso dos equipamentos.

Para participar do estudo, foram selecionados os indivíduos através de registros, prontuários eletrônicos e que atendessem aos seguintes critérios de inclusão:

- $\quad$ Ter recebido no período de janeiro a dezembro de 2017, dispositivos ou equipamentos de tecnologia assistiva do tipo cadeira de rodas manual ou motorizada, órteses e/ou próteses, independente de estar em uso contínuo ou de ter abandonado o dispositivo;

- Estar compreendido na faixa etária de 18 até 80 anos de idade.

Foram excluídos da pesquisa os sujeitos com incapacidade para comunicação, ou com déficit cognitivo que impossibilitasse a compreensão das perguntas, bem como pessoas que não apresentassem registro telefônico para contato. 
Pessoas que receberam equipamentos, residentes em outros municípios e sem continuidade de atendimentos no serviço de reabilitação também não puderam participar da pesquisa devido à dificuldade de deslocamento até o local do estudo.

A pesquisa respeita os princípios éticos referentes a estudos com seres humanos da Declaração de Helsinki (2000) e também da Resolução do Conselho Nacional de Saúde (CNS) no 466 de 2012. A pesquisa foi aprovada pelo Comitê de ética em pesquisa com seres humanos da Universidade Franciscana (UFN) por meio do CAAE no 91232118.8.0000.5306, parecer no 2903272 em 19 de setembro de 2018.

Os participantes da pesquisa assinaram o Termo de Consentimento Livre e Esclarecido (TCLE), em duas vias, sustentando uma consigo e a outra com o pesquisador. Foi fornecido o Termo de Confidencialidade (TC) a fim de garantir o sigilo da informação e identidade dos sujeitos participantes da pesquisa e indenização para casos de danos devidamente comprovados pela participação no estudo.

Todos os dados foram coletados por meio de um único pesquisador para evitar divergências durante a entrevista. Por meio de contato telefônico e disponibilização da agenda semanal de atendimento do serviço, os indivíduos foram convidados a participar da pesquisa. Tendo sido a pesquisa aprovada em setembro de 2018, a coleta de dados foi realizada nos meses de outubro e novembro do ano 2018 no serviço de reabilitação onde houve a dispensação das tecnologias assistivas.

Após a finalização das entrevistas, os dados encontrados foram tabulados em categorias e analisados de forma descritiva por meio do programa Microsoft Office Excel 2007 e Statistical Package for the Social Sciences(SPSS) versão 22.0. As categorias foram baseadas nas variáveis de cada questionamento da entrevista, tendo por finalidade simplificar as respostas e facilitar a discussão dos resultados semelhantes e dos divergentes.

Os dados foram submetidos a Estatística Descritiva, considerando nível de confiança de $95 \%$, significância $p<0,05$, tabela de referência cruzada para correlacionar as variáveis de abandono e treino, com resultados para razão de chance de evento ocorrer (odds ratio).

\section{RESULTADOS}

No período de janeiro a dezembro de 2017, para a faixa etária de 18 a 80 anos, foram dispensadas 117 próteses, 39 órteses e 175 cadeiras de rodas manuais e 69 cadeiras motorizadas, totalizando 400 TAs e 371 pessoas beneficiadas. Participaram desse estudo 31 pessoas que permanecem em atendimento ou acompanhamento no serviço.

Poucas pessoas participaram do estudo, devido ao não residirem no município do estudo e não possuírem mais o recurso do transporte para deslocar-se até o serviço, pois grande parte dos que receberam os recursos não possuem mais acompanhamento no serviço. Os dados demográficos em relação a idade e deficiência da amostra estão apresentados na Tabela 1.

Tabela 1. Caracterização sociodemográfica da amostra

\begin{tabular}{lccc}
\hline \multicolumn{1}{c}{ Variável } & $\mathbf{n = 3 1}$ & \% & DV \\
\hline 1. Masculino & 20 & 64,5 & 16,8 \\
2. Feminino & 11 & 35,4 & 19,4 \\
3. Deficiência Física & 31 & 100 & 0 \\
4. Deficiência Visual & 2 & 6,4 & 0,2 \\
5. Deficiência Intelectual & - & - & - \\
6. Deficiência Auditiva & - & - & - \\
\hline
\end{tabular}

$D V=$ desvio padrão

As 31 pessoas que responderam a entrevista, receberam um total de 38 equipamentos, sendo a maioria do sexo masculino, tendo mediana 50 anos, quartil 1 de 35 anos, idade máxima 78 anos, idade mínima 18 anos e quartil 3 de 59,5 anos (Figura 1). O número e o tipo de equipamentos recebido pelos entrevistados, estão apresentados na Figura 2.

Com relação ao processo de prescrição dos equipamentos, identificação profissional e a informação sobre o uso do recurso, estão apresentadas em percentil (Tabela 2).
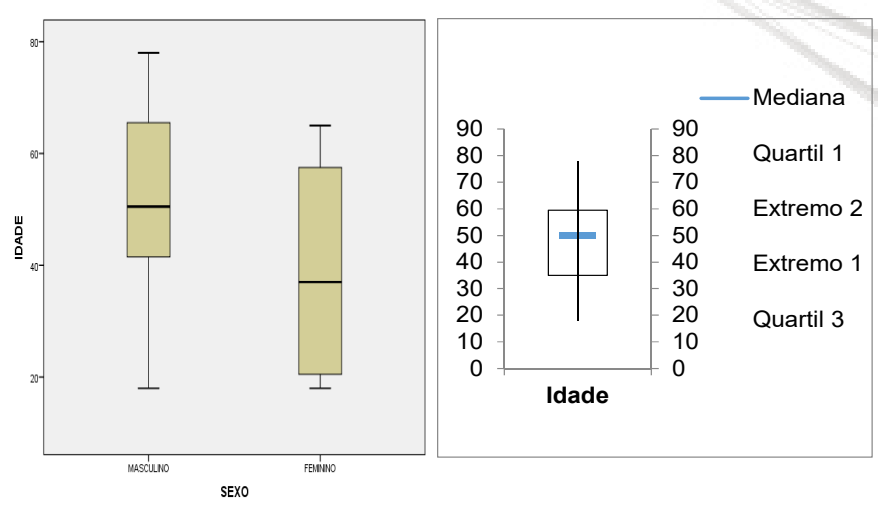

Figura 1. Correlação das idades e sexo da amostra através do diagrama de caixa

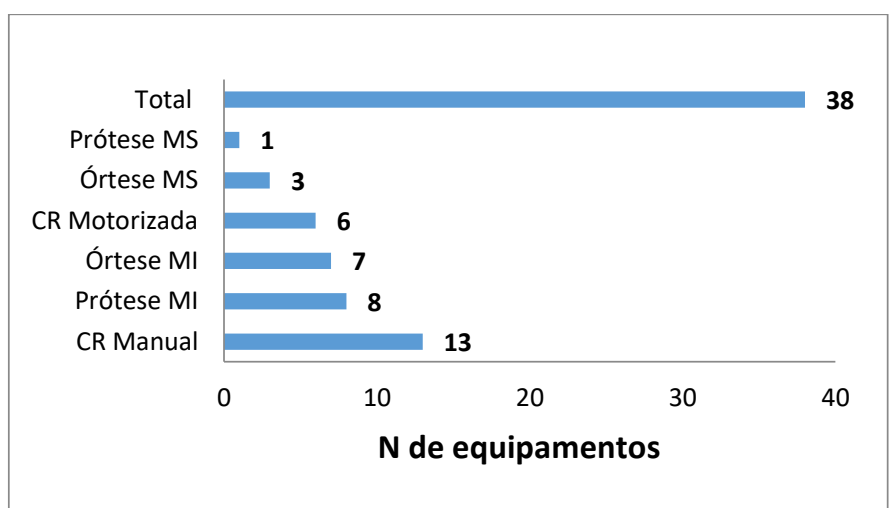

$C R=$ cadeira de rodas; $M I=$ membro inferior, $M S=$ membro superior

Figura 2. Tipo de equipamento mais recebido pelos participantes

Tabela 2. Informações sobre a prescrição das TAs

\begin{tabular}{lcccc}
\hline \multicolumn{1}{c}{ Variável } & $\mathbf{N = 3 1}$ & $\%$ & Media & DV \\
\hline Avaliação profissional & 28 & 93 & 1,12 & 0,33 \\
Sim & 3 & 7 & 1 & - \\
Não Soube & & & & \\
Profissional que avaliou & 20 & 64,5 & 1,08 & 0,27 \\
Fisioterapeuta & 2 & 6,4 & 1 & - \\
Ortopedista & 2 & 6,4 & 1 & - \\
Terapeuta Ocupacional & 7 & 22,5 & 1,14 & 0,37 \\
Não soube & 27 & 87,1 & 1,9 & 0,29 \\
Recebeu informação sobre o recurso e suas funções & \\
Sim & 4 & 12,9 & 1 & - \\
Não & \multicolumn{5}{c}{}
\end{tabular}

DV=Desvio Padrão. Dados gerados por meio da estatística descritiva de frequência

Para verificar a satisfação do usuário com o equipamento e a habilitação recebida para uso dos recursos, estão os resultados dispostos em percentil (Tabela 3), conforme encontrado pela entrevista. Os entrevistados responderam qual sua percepção sobre a utilização do equipamento que receberam, os resultados estão apresentados por aspectos positivos e negativos na Tabela 4.

Os indivíduos participantes responderam quais as contribuições que esperavam ter dos equipamentos antes de o terem recebido e de usar. Os resultados estão apresentados em ordem decrescente de benefício (Figura 3). 
Tabela 3. Dados sobre a satisfação e a habilitação para uso do equipamento

\begin{tabular}{lcccc}
\hline \multicolumn{1}{c}{ Variável } & $\mathbf{N = 3 1}$ & $\%$ & Media & DV \\
\hline Satisfação com o produto & 26 & 83,8 & 1 & - \\
Sim & 5 & 16,2 & 2 & - \\
Não & & & & \\
Recebeu treino & 23 & 74,1 & 1,16 & 0,37 \\
Sim & 8 & 25,8 & 1,6 & 0,54 \\
Não &
\end{tabular}

DV=Desvio Padrão. Dados gerados por meio da estatística descritiva de frequência

Tabela 4. Dados sobre a utilização do equipamento pelo paciente

\begin{tabular}{lcccc}
\hline \multirow{2}{*}{ Variável } & \multicolumn{2}{c}{ N=31 } & \multicolumn{2}{c}{$\%$} \\
& Sim & Não & Sim & Não \\
\hline Equipamento pesado & 8 & 23 & 25,8 & 74,1 \\
Constrangimento no uso & 2 & 29 & 6,4 & 93 \\
Dificuldade para uso & 8 & 23 & 25,8 & 74,1 \\
Formação de lesões & 12 & 19 & 38,7 & 61,2 \\
Utiliza o recurso atualmente & 27 & 4 & 87 & 12,9 \\
Aparência Atrativa & 21 & 10 & 67,7 & 32,2 \\
Confortável & 15 & 16 & 48,3 & 51,6 \\
Sente-se bem no uso & 22 & 9 & 70,9 & 29,1 \\
Contribuição na Independência & 25 & 6 & 80,6 & 19,3 \\
Utiliza o recurso atualmente & 27 & 4 & 87 & 12,9 \\
\hline
\end{tabular}

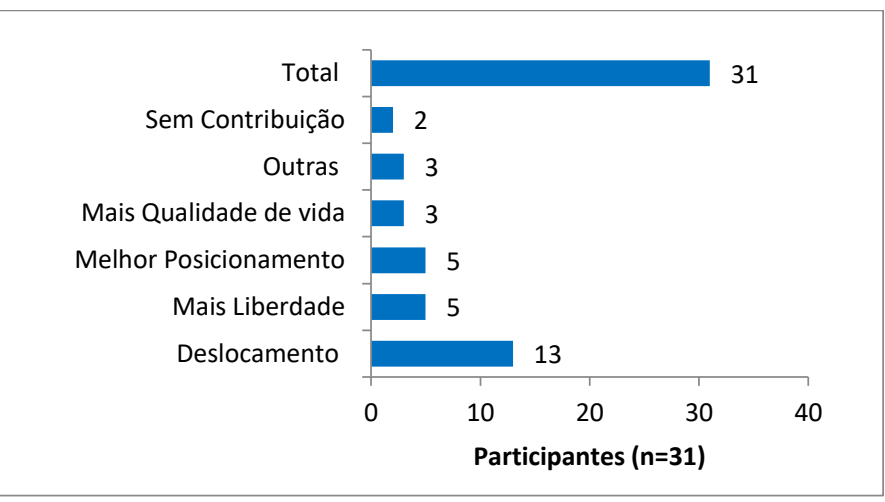

Figura 3. Benefícios esperados antes de utilizar o equipamento

Após utilizar o recurso, os indivíduos relataram os benefícios que observaram após fazer uso do recurso (Figura 4).

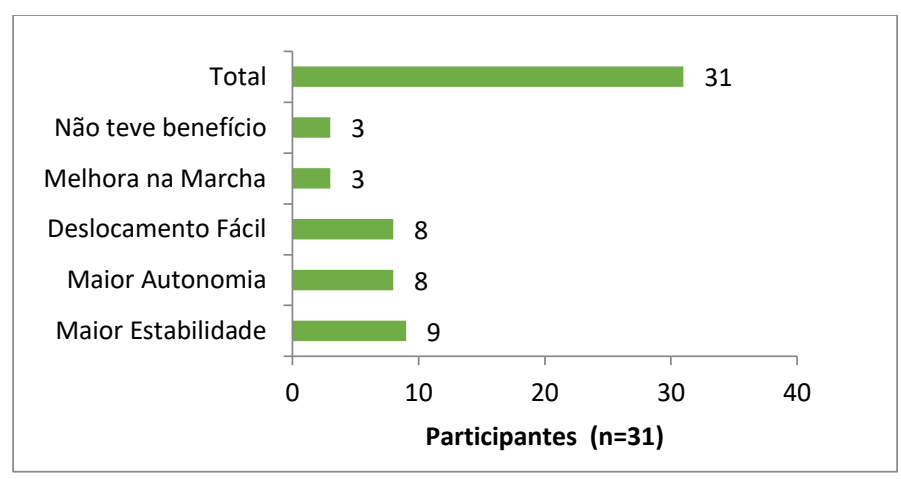

Figura 4. Benefícios observados após ter utilizado o recurso

Com relação à assiduidade e o abandono do equipamento, a pesquisa encontrou que dos 38 equipamentos, 18 (47,3\%) foram abandonados e $20(52,6 \%)$ continuam sendo utilizados. Dos 18 equipamentos que foram abandonados, 8 correspondem a cadeiras de rodas, 5 órteses e 5 próteses, esses recursos entraram em desuso conforme os próprios indivíduos por alguns motivos, dentre eles a formação de lesões, dor no uso do equipamento, dificuldade para saber utilizar, aparência desagradável e por estarem armazenados na oficina ortopédica, pois não se adequaram as necessidades do indivíduo que o recebeu.

Comparando a variável Abandono ou Em Uso com a variável Treino (se recebeu ou não treino para uso) por meio de Tabela Cruzada no SPSS, obteve-se a razão de chances - odds Ratio (OR) (Tabela 5).

Tabela 5. Estimativa de risco comparando as variáveis situação e treino

\begin{tabular}{lrrrr}
\hline SEXO & Valor & Inferior & Superior \\
\hline Masculino & $\begin{array}{r}\text { TREINO (Sim/Não) } \\
\text { OR }\end{array}$ & 3,125 & 0,474 & 20,58 \\
& SITUAÇÃO = Abandono & & & \\
OR & 1,944 & 0,562 & 6,733 \\
& SITUAÇÃO = Em Uso & & & \\
OR & 0,622 & 0,31 & 1,25 \\
Feminino & No de Casos Válidos & 20 & & \\
& TREINO (Sim/Não) & 1,25 & 0,806 & 1,938 \\
& SITUAÇÃO = Em Uso & & & \\
OR & 0,4 & 0,187 & 0,855 \\
& No de Casos Válidos & 11 & & \\
\hline OR= odds ratio; Intervalo de confiança de 95\% & & &
\end{tabular}

\section{DISCUSSÃO}

Os resultados do estudo mostram que a maioria dos participantes que utilizam cadeira de rodas, órtese ou prótese estão na faixa etária adulta e idosa. Alguns estudos já evidenciam que a idade, relacionada a fatores de risco como Diabetes Mellitus, Hipertensão Arterial, acidentes traumáticos e doenças vasculares, tem sido grande causa para o aumento no número de amputações e outras complicações clínicas. ${ }^{12,13}$

Grande parte das pessoas que utilizam uma prótese necessitam desse equipamento em consequência de amputações e por vezes pela má formação congênita dos membros. Já a cadeira de rodas e as órteses, geralmente são utilizadas no tratamento das deficiências congênitas ou adquiridas para favorecer o deslocamento e ganho de amplitude de movimento, respectivamente. ${ }^{14}$

Estudos destacam que as cadeiras de rodas são comumente utilizadas em casos de lesões traumáticas e medulares, sendo que o assento e o encosto desse recurso merecem ampla atenção para que não ocasionem lesões. Já as próteses, principalmente de membro inferior, usadas para reestabelecer a marcha, necessitam de uma avaliação adequada, pois ao ocasionar a marcha ceifante no paciente, provocará possível desgaste ou desvios posturais. ${ }^{11}$

Nesse trabalho, dentre os equipamentos dispensados pelo serviço de reabilitação, a cadeira de rodas manual foi a mais dispensada. Esta, socialmente vem sendo um equipamento amplamente dispensado pelo SUS, devido a sua alta usabilidade e funcionalidade, possibilitando ao indivíduo um meio de locomoção, para participação social e integração comunitária. ${ }^{12}$

No entanto, os dados mostraram que duas cadeiras de rodas tiveram abandono de uso, o que pode estar relacionado a dificuldade de utilização no ambiente doméstico e comunitário de convivência. Esse dado pode ser devido ao ambiente apresentar algumas barreiras, interferindo na facilitação que os recursos poderiam permitir ou ainda por falta de informações quanto ao uso e treino.

Seguida da cadeira de rodas manual, o segundo equipamento mais dispensado foram as próteses de membro inferior. Essas próteses apresentam maior taxa de usabilidade e adaptação quando comparadas a de membro superior, pois os indivíduos com amputação de membro superior mostram-se pouco satisfeitos com o grau de 
tecnologia das próteses ofertadas, abandonando em maior número e procurando pouco esse recurso. ${ }^{15}$ Isso confirmou-se no presente estudo quando os resultados mostraram que foi dispensada apenas uma prótese de membro superior entre os participantes e a mesma foi abandonada.

A prótese de membro superior abandonada, segundo o paciente não apresentava uma aparência agradável. Tratando-se de próteses estéticas de membro superior, alguns estudos referem que essas próteses não proporcionam funções ativas de movimento, tendo apenas favorecimento no quesito estético, e quando esse equipamento não possui um aspecto agradável acaba por não cumprir seu objetivo. ${ }^{16}$

Sobre a prescrição dos equipamentos, o estudo evidenciou que 93\% dos participantes foram avaliados por profissionais da saúde, em sua maioria Fisioterapeuta, Ortopedista e Terapeuta Ocupacional, de modo que esses profissionais atuam de forma ampliada nos cuidados de reabilitação. Nesse processo, a maioria dos participantes receberam informações sobre os recursos, seus componentes e funções, além da importância do uso para o tratamento. Pode-se analisar que, essa atenção adequada no momento da prescrição pode atuar de forma positiva para que os usuários sejam conscientizados da importância desse equipamento no tratamento.

Em estudo ${ }^{11}$ a falta de informação/conscientização tem sido relatada com mais frequência como um motivo de abandono de TAs, o que pode estar relacionado com os dados dessa pesquisa, a qual mostraram alguns casos que relataram não receber orientação, bem como situações de abandono.

Por meio desse trabalho, foi possível verificar que a maioria dos participantes entrevistados $(83,8 \%)$ estão satisfeitos com o recurso recebido, uma vez que $67,7 \%$ referem boa aparência dos equipamentos, $70 \%$ sente-se bem quando estão em uso e $74 \%$ receberam treinamento para saber utilizar adequadamente os dispositivos. A orientação adequada durante o processo de prescrição e de treino com o paciente, além de reforçar o benefício do equipamento contribui positivamente para que não ocorra esse abandono.

Além disso, a expectativa quando ao uso dos recursos contribui de forma positiva para a adesão, pois muitas vezes experiências mal sucedidas anteriormente, negação da deficiência e o sentimento de culpa podem atuar como fatores que dificultam a adesão das TAs. ${ }^{17}$

A boa adesão de muitos pacientes às TAs também pode estar relacionada as suas contribuições para o tratamento e para a saúde. Dentre as contribuições mais informadas nesse estudo, estão a facilitação do deslocamento e a maior estabilidade proporcionada por esses recursos.

Percebe-se que essas contribuições dizem respeito às cadeiras de rodas, órteses e próteses de membro inferior, devido ao fato de favorecerem o deslocamento de forma mais independente. Além dessas funções, essas tecnologias citadas possuem outras colaborações importantes ao tratamento de reabilitação, como a correção de padrões anormais de posicionamento, favorecimento da aplicação da força muscular, do equilíbrio e da amplitude de movimento. 12

Contudo, ressalta-se que apesar de estar satisfeito com o equipamento recebido, cerca de $30 \%$ das pessoas relataram alguma dificuldade para utilização, podendo estar relacionado ao peso do equipamento, o quão confortável é e também a formação de lesões. Outro estudo semelhante já trouxe evidência de que o peso, formação de lesão e dor, além de outras dificuldades contribuem para a pouca efetividade da prescrição e uso das tecnologias assistivas. ${ }^{11}$

A Tabela 5 trouxe a análise comparando a situação de abandono com o fato de ter recebido treino no momento da entrega do equipamento. Pode se sugerir que as pessoas que não receberam treino têm maior risco de abandonar seus equipamentos. Estudo semelhante, destaca a importância para o treino de instrução, além do acompanhamento do uso da TA, para que ocorra maior eficácia do equipamento e por consequência maior risco de abandono. ${ }^{18}$
Desde o processo de prescrição, cabe ao profissional avaliar o paciente de forma adequada e ampliada para atender as necessidades, sendo assessorado de uma equipe multiprofissional capacitada nesse quesito avaliativo. Conforme este estudo, apenas 13 pessoas tiveram satisfação total na prescrição e 14 ainda passam por processo de ajuste, sendo considerada na análise essa situação como abandono do equipamento, pois o mesmo não está sendo usado estando na oficina ortopédica.

O abandono dos equipamentos teve um representativo quantitativo nessa pesquisa, justifica-se que essa situação não deveria ocorrer, uma vez que, o equipamento foi prescrito a partir de um objetivo importante no tratamento e o usuário que permanece em acompanhamento no serviço, não passou por reavaliação ou substituição de dispositivo.

Conforme ressaltado, o serviço de reabilitação possui a responsabilidade por avaliar, experimentar e treinar o uso dos equipamentos de Tecnologia Assistiva, além de manter acompanhamento periódico com o paciente e seus equipamentos. ${ }^{19}$

Dessa forma, o acompanhamento periódico do equipamento, além de evitar o abandono, melhora a satisfação do paciente, contribui para o sucesso das prescrição e aumenta a responsabilização do indivíduo para cuidar de forma adequada do dispositivo, para que possa manter o benefício que lhe proporciona.

Nesse estudo, parte dos participantes permanece em contato com o serviço de reabilitação, realizando atendimento ou mantendo acompanhamento para os equipamentos, em contrapartida outros não.

Avalia-se que uma das fragilidades do estudo está em não conseguir contato com as demais pessoas que receberam os 362 equipamentos, pois a falta de continuidade dos atendimentos no serviço e a inexistência do recurso de transporte através dos municípios distantes, dificultaram a participação desses outros indivíduos na pesquisa, onde possivelmente encontrar-se-ia maiores motivações para adesão ou outros motivos de abandono das tecnologias assistivas.

\section{CONCLUSÃO}

Partindo-se da análise desse estudo, destaca-se que adultos e idosos são o maior público usuário de cadeira de rodas, órteses e próteses, estando satisfeitos com os equipamentos recebidos e com as contribuições ao tratamento. As pessoas que relataram dificuldade no uso, estes o atribuem ao peso, a formação de lesões, a dor e a aparência.

Os profissionais que fazem a prescrição dos dispositivos são Fisioterapeutas, Terapeutas Ocupacionais e Ortopedistas, tendo enfoque na prescrição, sendo que na entrega do equipamento o treino de habilitação e os ajustes para as inadequações que possam existir são realizados na maioria dos casos, fazendo com que o usuário sintase mais satisfeito e também contribuindo para que não haja abandono do equipamento.

Porém, mesmo que a maioria dos usuários relataram estar satisfeitos, existe uma parcela de pessoas insatisfeitas que não consegue fazer uso dos equipamentos devido aos motivos anteriormente citados.

Por fim, observou-se que a adesão das tecnologias foi mais prevalente, do que o processo de abandono, pois mesmo estando em processo de ajuste há permanência e interesse em utilização dos equipamentos por parte dos entrevistados, devido as contribuições ao tratamento e a saúde, sendo o número de abandono bem abaixo do esperado.

Pondera-se que o quantitativo de abandono, poderia ter sido bem acima do apresentado, caso os usuários residentes de outros municípios pudessem ter participado do estudo, pois os mesmos por estarem sem acompanhamento, possivelmente apresentam grandes dificuldades em utilizar os recursos sem o acompanhamento adequado e sem apoio da rede de saúde local. 
Compreende-se que o processo de alta paciente do serviço, deve ser realizado por meio da contra referência ao local de origem, a fim de que o usuário possa se manter em acompanhamento periódico e retornando ao ambiente da dispensação, caso seja necessário a troca ou ajuste nos equipamentos para um melhor uso e evitando assim o abandono dos recursos.

\section{AGRADECIMENTOS}

A todos que contribuíram com o compartilhamento de conhecimento, disponibilização do ambiente para pesquisa e demais situações que favoreceram o desenvolvimento desse estudo.

\section{REFERÊNCIAS}

1. Organização Mundial de Saúde. Relatório mundial sobre a deficiência. São Paulo: SEDPcD; 2012.

2. Oliveira LMB. Cartilha do Censo Demográfico de 2010. Brasília (DF): SDH-PR/SNPD; 2012.

3. Gaudenzi P, Ortega F. Problematizando o conceito de deficiência a partir das noções de autonomia e normalidade. Ciên Saúde Colet. 2016;21(10):3061-70. DOI: http://dx.doi.org/10.1590/1413 812320152110.16642016

4. Brasil. Lei n. 13.146 de 6 de julho de 2015. Institui a Lei Brasileira de Inclusão da Pessoa com Deficiência (Estatuto da Pessoa com Deficiência). Diário Oficial da República Federativa do Brasil, Brasília (DF): 2015 Jul 7; Seção 1:2-11.

5. Bersch R. Introdução à tecnologia assistiva. Porto Alegre: CEDI; 2013.

6. Silva MGC. Causas de abandono de dispositivos de tecnologia assistiva [Monografia]. Brasília: Universidade de Brasília; 2016.

7. Parant A, Schiano-Lomoriello S, Marchan F. How would I live with a disability? Expectations of bio-psychosocial consequences and assistive technology use. Disabil Rehabil Assist Technol. 2017;12(7):681-5. https://doi.org/10.1080/17483107.2016.1218555

8. Federici S, Meloni F, Borsci S. The abandonment of assistive technology in Italy: a survey of National Health Service users. Eur J Phys Rehabil Med. 2016;52(4):516-26.

9. Ferreira $A B H$. Novo Aurélio Século XXI: o dicionário da língua portuguesa. Rio de Janeiro: Nova Fronteira; 1999.

10. Scherer MJ, Federici S. Why people use and don't use technologies: Introduction to the special issue on assistive technologies for cognition/cognitive support technologies. NeuroRehabilitation. 2015;37(3):315-9. DOI: https://doi.org/10.3233/NRE-151264

11. Costa CR, Ferreira FMRM, Bortolus MV, Carvalho MGR Dispositivos de tecnologia assistiva: fatores relacionados ao abandono. Cad Ter Ocup UFSCar. 2015; 23(3):611-24. DOI: https://doi.org/10.4322/0104-4931.ctoAR0544

12. Carvalho FS, Kunz VC, Depieri TZ, Cervelini R. Prevalência de amputação em membros inferiores de causa vascular: análise de prontuários. Arq Ciênc Saúde. 2005;9(1):23-30. DOI: https://doi.org/10.25110/arqsaude.v9i1.2005.215

13. Brasil. Ministério da Saúde. Diretrizes de atenção à pessoa amputada. Brasília (DF): Ministério da Saúde; 2013.

14. Santos KT, Soglia L. Sustentando-se nas lesões: problemas ocasionados pela biomecânica incorreta de próteses/órteses e cadeira de rodas. Rev EFDeport. 2012:16(164).

15. Feldhaus LC. Estudo e otimização do envoltório externo das próteses de antebraço [Monografia]. Pato Branco: Universidade Tecnológica Federal do Paraná; 2017.

16. Tavares PC, Tormes LH, Araújo RA, Torres BR. Intervenção protética em amputação de membros superiores (MMSS). In: X Encontro de Iniciação à Docência; 2000; Recife. Anais. Recife: Universidade Federal do Pernambuco; 2000.
17. Bittencourt ZZLC, Cheirad DC, Montilha RCl, Gasparetto MERF. Expectativa quanto ao uso de tecnologia assistiva. Jorsen. 2016;16(S1):492-6.

18. Lustosa LP, Andrade MAP, Araújo MRN, Bonolo PF, Campos TVO, Araújo VL. Uso terapêutico de tecnologias assistivas: direitos das pessoas com deficiência e habilidade física e motora. Belo Horizonte: Nescon; 2015.

19. Scatolim RB, Santos JEG, Landim PC, Toledo TG, Fermino SCM, Cardozo $D$, et al. Legislação e tecnologias assistivas: aspectos que asseguram a acessibilidade dos portadores de deficiências. Rev NEaD-Unesp. 2016;2(1):227-48. 